Enferm Bras 2020;19(2):130-7

https://doi.org/10.33233/eb.v19i2.3118

\title{
ARTIGO ORIGINAL \\ Os motivos de não-adesão ao exame preventivo de câncer de colo uterino e ações educativas em uma região marajoara
}

Elisângela da Silva Ferreira, M.Sc. *, Sheila Barbosa Paranhos, M.Sc. *, Edficher Margotti, D.Sc. Sandra Moura da Silva**, Simone de Carvalho Barboza***

*Enfermeiras, Docentes do Curso de Enfermagem da Universidade Federal do Pará, ${ }^{* *}$ Enfermeira, Prefeitura Municipal de Portel, Pará, ${ }^{* * *}$ Enfermeira, Prefeitura Municipal de Salvaterra, Pará

Recebido em 30 de julho de 2019; aceito em 6 de abril de 2020.

Correspondência: Elisângela da Silva Ferreira, Rua Caripunas 1220/408 edifício Debora, Jurunas, 66033230 Belém PA

Elisângela da Silva Ferreira: licalipe8@yahoo.com.br

Sheila Barbosa Paranhos: paranhos@ufpa.br

Edficher Margotti: edficher@ufpa.br

Sandra Moura da Silva: sandraamoura@yahoo.com.br

Simone de Carvalho Barboza: simone barbosa@yahoo.com.br

\section{Resumo}

No Brasil, o câncer de colo do útero é o terceiro tumor mais frequente na população feminina, sendo o primeiro mais incidente na Região Norte. Estudos nos revelam que a baixa adesão ao exame Preventivo do Câncer de Colo de Útero (PCCU) se acentua entre as mulheres das regiões mais pobres do país. Este estudo tem como objetivo descrever os fatores da não-adesão ao PCCU, incentivando-as por meio de ações educativas à prevenção do câncer do colo uterino. Estudo do tipo descritivo, prospectivo, com abordagem quantitativa, desenvolvido no município de Portel, localizado na mesorregião do Marajó, no estado do Pará, com 250 mulheres, residentes na área de cobertura da Estratégia Saúde da Família (ESF) da Castanheira e do Centro de Referência da Zona Rural que atende a população ribeirinha residente às margens dos três rios que banham o município, após busca ativa realizada pelos Agentes Comunitários de Saúde (ACS), em suas áreas de abrangência. Posteriormente ao preenchimento do instrumento de coleta de dados, as mulheres eram convidadas a participar da ação educativa. Os dados coletados foram analisados através de estatística descritiva. A maioria das participantes já havia realizado o exame, entretanto uma parcela já havia feito há mais de 2 anos. Os motivos de não-adesão (que nunca realizaram ou fizeram há mais de dois anos) se assemelham entre as moradoras da área urbana e rural, dentre eles: dificuldade de acesso ao local de realização do exame e medo, vergonha/constrangimento para coletar o material, falta de interesse, não saber que deveria realizar o exame, falta de material e a proibição do marido. O planejamento das ações educativas levou em consideração esses motivos e a necessidade de orientações e esclarecimentos. O estudo possibilitou demonstrar a importância da educação em saúde, através de ações educativas, desenvolvidas e pensadas de acordo com a realidade de cada localidade.

Palavras-chave: saúde da mulher, educação em saúde, teste de Papanicolau, Enfermagem.

\footnotetext{
Abstract

The reasons for non-adhesion to screening test of cervical cancer and educational actions in a Marajoara region

In Brazil, cervical cancer is the third most frequent tumor in the female population, the first being more incident in the Northern Region. Studies show that the low adherence to the Prevention Test of Cervical Cancer (PTCC) is increasing among women in the poorest regions of the country. This study aimed to describe the factors of non-adherence to the PTCC, encouraging them through educational actions to prevent cervical cancer. A descriptive, prospective study with a quantitative approach, developed in the municipality of Portel, located in the Marajó meso-region, in the state of Pará, with 250 women who lived in the Family Health Strategy (ESF) area of
} 
Castanheira and the Center of reference of the rural area that serves the resident riverside population on the banks of the three rivers that bathe the municipality, after an active search carried out by the Community Health Agents (ACS), in their areas of coverage. After completing the data collection instrument, the women were invited to participate in the educational action. The collected data were analyzed through descriptive statistics. Most of the participants have already done the test, however a portion had already done for more than 2 years. The reasons for non-adherence (never have done or have done two years ago) are similar among urban and rural dwellers, among them: difficulty in accessing the local of test screening and fear, shame/embarrassment to collect the material, lack of interest, not knowing that have to do the test, lack of material and the prohibition of the husband. The planning of educational actions considered these reasons and the need for orientation and clarification. The study made it possible to demonstrate the importance of health education, through educational actions, developed and thought according to the reality of each locality.

Keywords: women's health, health education, Papanicolaou test, Nursing.

\section{Resumen}

\section{Las razones para la no adhesión al examen preventivo del cáncer de cuello uterino y las acciones educativas en una región Marajoara}

En Brasil, el cáncer de cuello uterino es el tercer tumor más frecuente en la población femenina, siendo el primer incidente más frecuente en la Región Norte. Los estudios revelan que la baja adherencia al examen preventivo de cáncer de cuello uterino (PCCU) se acentúa entre las mujeres en las regiones más pobres del país. Este estudio tiene como objetivo describir los factores de no adherencia a la PCCU, incentivándolas a través de acciones educativas para prevenir el cáncer de cuello uterino. Estudio descriptivo prospectivo con enfoque cuantitativo, desarrollado en el municipio de Portel, ubicado en la mesorregión de Marajó, en el estado de Pará, con 250 mujeres, que residen en el área de cobertura de la Estrategia de Salud Familiar (FSE) de Castanheira y Centro de Referencia de la Zona Rural que atiende a la población ribereña que reside en las orillas de los tres ríos que bañan al municipio, después de una búsqueda activa realizada por los Agentes Comunitarios de Salud (ACS), en su áreas de cobertura. Después de cumplimentar el instrumento de recolección de datos, las mujeres fueron invitadas a participar de la acción educativa. Los datos recopilados se analizaron mediante estadística descriptiva. La mayoría de las participantes ya habían realizado el examen, sin embargo, una parte lo había hecho hace más de 02 años. Las razones de la no adherencia (que nunca realizaron o hicieron hace más de dos años) son similares entre los residentes de las áreas urbanas y rurales, entre ellas: dificultad para acceder al lugar del examen y miedo, vergüenza/vergüenza para recoger el material, falta de interés, no saber que se debe realizar el examen, falta de material y la prohibición del marido. La planificación de las acciones educativas tuvo en cuenta estos motivos y la necesidad de orientación y aclaración. El estudio permitió demostrar la importancia de la educación para la salud, a través de acciones educativas, desarrolladas y diseñadas de acuerdo con la realidad de cada lugar.

Palabras-clave: salud de la mujer, educación en salud, prueba de Papanicolaou, Enfermería.

Introdução

O câncer do colo do útero caracteriza-se pela replicação exagerada do epitélio de revestimento do órgão que compromete o tecido subjacente, podendo acometer outras estruturas próximas ou distantes. O principal fator de risco para o desenvolvimento de lesões intraepiteliais de alto grau precursoras do câncer uterino e do câncer do colo do útero, propriamente dito, é a infecção pelo papilomavírus humano (HPV) [1].

Para o Brasil, estimam-se 16.370 casos novos de câncer do colo do útero para cada ano do biênio 2018-2019, com um risco estimado de 15,43 casos a cada 100 mil mulheres, ocupando a terceira posição, sendo o primeiro mais incidente na Região Norte, com 25,62/100 mil [2].

A principal estratégia para reduzir a alta incidência e mortalidade do câncer do colo do útero é o seu rastreamento através do exame Preventivo do Câncer de Colo de Útero (PCCU), o Papanicolau, porém a adesão a esse exame ainda está distante da cobertura preconizada [3]. Estudos revelam que a baixa adesão ao PCCU se acentua entre as mulheres das regiões mais pobres do país, tornando-as mais vulneráveis a este tipo de câncer, evidenciando que baixas condições socioeconômicas estão relacionadas a maiores incidências ao câncer do colo do útero [4]. 
Casarin e Piccoli [5] afirmam que existe uma associação entre o câncer de colo uterino e o baixo nível socioeconômico em todas as regiões do mundo. Para eles, os grupos mais vulneráveis estão onde existem barreiras de acesso à rede de serviços de saúde, para detecção e tratamento da patologia e de suas lesões precursoras, advindas das dificuldades econômicas e geográficas, insuficiência de serviços e por questões culturais, como medo, desconsideração de sintomas importantes e preconceito. Vale ressaltar que o local de realização deste estudo apresenta um dos Índices de Desenvolvimento Humano (IDH) mais baixos do país [6].

Com um levantamento de dados, realizado na Secretaria de Saúde do município de Portel, identificou-se que no período de 2010 a 2016 registraram-se 49 casos novos de câncer do colo do útero (média de 8,1 casos por ano), além disso, percebeu-se que este número poderia não corresponder à realidade por serem registrados apenas os casos acompanhados pelo TFD (Tratamento Fora Domicílio), não sendo contabilizados, nesses registros, os tratamentos realizados sem TFD e os casos não tratados.

Com base nisso, surgiu o interesse em conhecer a realidade do município de Portel no que concerne a adesão ao exame preventivo do câncer do colo uterino e os fatores relacionados à sua não-adesão por um grupo de mulheres, pois o desenvolvimento de estudos que busquem identificar os fatores associados à realização do exame preventivo é importante para subsidiar intervenções qualificadas e efetivas, capazes de impactar na incidência do câncer de colo de útero em subgrupos vulneráveis [7].

Para tanto, este estudo tem como objetivo descrever os fatores da não-adesão ao exame Papanicolau das mulheres residentes no Marajó, incentivando-as por meio de ações educativas a prevenção do câncer do colo uterino.

Estudo do tipo descritivo, prospectivo, com abordagem quantitativa, desenvolvido no município de Portel, localizado na Mesorregião do Marajó, no estado do Pará, realizado com 250 mulheres residentes na área de abrangência da Estratégia Saúde da Família (ESF) da Castanheira, localizada na zona urbana, e no Centro de Referência da Zona Rural, que atende a população ribeirinha residente às margens dos três rios que banham o município.

Antes da coleta dos dados, os Agentes Comunitários de Saúde (ACS) (no total de 25 profissionais) participaram de uma reunião para demonstração da metodologia do estudo. Os dados foram coletados no período de outubro a novembro de 2017, em uma busca ativa realizada pelos ACS do município, em suas áreas de abrangência, utilizando um questionário contendo questões sobre o perfil socioeconômico e demográfico das participantes e quanto à adesão e regularidade na realização do PCCU.

Após aceite e assinatura do Termo de Consentimento Livre e Esclarecido (TCLE), as participantes preenchiam o instrumento de coleta dos dados e posteriormente eram convidadas a participar da ação educativa. As mulheres que buscaram, espontaneamente, atendimento na ESF Castanheira e Centro de Referência da Zona Rural, que atenderam aos critérios de inclusão, também foram convidadas a participar do estudo, seguindo as mesmas etapas das que receberem a visita domiciliar.

Os dados coletados foram analisados através de estatística descritiva, com base nos números absolutos e relativos, de forma evolutiva em gráficos e tabelas.

O projeto deste estudo passou por apreciação da Secretaria Municipal de Saúde de Portel para autorização e, posteriormente, por avaliação e análise do Comitê de Ética e Pesquisa (CEP) do Instituto de Ciências da Saúde (ICS) da Universidade Federal do Pará, sendo aprovado através do CAAE no 69890017.0.0000.5634. A pesquisa foi realizada com base na resolução $n=466 / 2012$, do Conselho Nacional de Saúde, de acordo com as diretrizes e normas regulamentadoras sobre pesquisa envolvendo seres Humanos.

Resultados

Em relação ao perfil socioeconômico, observou-se que a maioria das participantes da zona rural tinha entre 20 e 29 anos (52/150), possuía ensino fundamental incompleto (54/150), eram lavradoras (54/150), viviam em união estável (62/150), e tinham renda de menos de um salário mínimo (139/150). Na ESF da zona urbana, identificamos que o perfil das participantes 
se assemelha ao das mulheres residentes na zona rural, em sua maioria, estavam na faixa etária de 20 a 29 anos (45/100), porém assim como na zona rural, apresentavam baixa escolaridade, nível fundamental incompleto (47/100), ocupavam-se das atividades do lar (62/100), viviam em união estável (52/100) e tinham renda de menos de um salário mínimo (88/100).

Em relação à realização do PCCU pelas mulheres residentes na zona rural, o presente estudo aponta que, do total de participantes, a maioria já havia realizado o exame (99/150), destas, 30/99 afirmaram ter feito o mesmo entre um e dois anos e 23/99 o realizaram há mais de três anos. Entretanto, 34\% das mulheres participantes do estudo nunca realizaram o exame e das que já haviam feito PCCU, 28\% estavam há mais de 02 anos sem fazer. Na zona urbana a grande maioria das participantes da pesquisa já realizou o exame $(90 / 100), 10 \%$ das participantes nunca realizaram (10/100), entretanto, das que já haviam realizado, 22\% o fizeram há mais de 02 anos.

A tabela I demonstra os motivos para não-adesão ou irregularidade na realização do PCCU pelas mulheres que nunca realizaram o exame e as que o realizaram há mais de 02 anos, consideradas irregulares nesta pesquisa, com base nas recomendações do Ministério da Saúde, residentes na zona rural e urbana de Portel. Observa-se que os motivos se assemelham entre as moradoras da área urbana e rural. Dentre as participantes residentes na zona rural, a maioria aponta que o motivo tem relação com a dificuldade de acesso ao local de realização do exame (22/93), seguido de vergonha/constrangimento para coletar o material (20/93). A maioria das participantes da zona urbana descreve que foi por falta de interesse (12/32) e por não saber que deveria realizá-lo $(07 / 32)$.

Tabela I - Descrição dos motivos para não-adesão ou irregularidade na realização do PCCU pelas mulheres residentes na zona rural e urbana do município de Portel/PA, 2017.

\begin{tabular}{llllll}
\hline & & Zona rural & \multicolumn{2}{c}{ Zona urbana } \\
\hline Variável & Descrição & $\mathrm{N}$ & $\mathrm{F}(\%)$ & $\mathrm{N}$ & $\mathrm{F} \mathrm{( \% )}$ \\
\hline & Não teve interesse & 9 & 5,4 & 12 & 37,50 \\
& Não sabia que deveria realizar & 6 & 20,4 & 07 & 21,9 \\
& Medo & 0 & 17,2 & 00 & 0,00 \\
& Vergonha/Constrangimento & 2 & 21,5 & 01 & 3,1 \\
& Difícil acesso ao local de coleta & 3 & 23,6 & 04 & 12,5 \\
& Proibição do marido/companheiro & 3 & 3,2 & 00 & 0,00 \\
& Falta de material & 3 & 8,7 & 08 & 25,00 \\
\hline total & & 100,00 & 32 & 100,00 \\
\hline
\end{tabular}

Fonte: Instrumento de coleta de dados, Portel/PA, 2017.

Os resultados apresentados serviram como base para a realização de ações educativas, especialmente quanto aos motivos da não-adesão do PCCU, objetivando sanar as dúvidas apresentadas pelas mulheres e desmistificar o exame preventivo de câncer de colo uterino.

Elaborou-se uma dinâmica com imagens e frases representando os motivos citados como barreira para a realização do exame, sendo os principais: medo, não sabia que tinha que fazer, vergonha e constrangimento, falta de material, difícil acesso e proibição pelo marido. Com intuito de demonstrar as possíveis consequências da não-adesão ao exame, foram construídos dois caminhos que levavam à "doença" ou, se realizado, aos benefícios e hábitos necessários para prevenir ou diagnosticar precocemente o câncer do colo do útero, como: higiene intima, realizar o PCCU regulamente, uso de camisinha, evitar múltiplos parceiros, expressado ao final deste caminho como "corpo saudável".

Essas ações tiveram como tema principal "prevenção do colo do útero e importância do exame PCCU" e foram distribuídas em etapas, após convite formal às participantes: a) a primeira consistiu em exposição oral com esclarecimentos quanto ao tema com o auxílio de aparelho multimídia, utilizando-se linguagem simples e imagens para melhor compreensão do tema; b) na segunda etapa realizou-se uma dinâmica, intitulada "ultrapassando obstáculos a caminho de um corpo saudável". Nessa dinâmica dividiram-se as participantes em dois grupos, as que se encontravam sentadas no lado direito do auditório e as que se estavam no esquerdo. Para cada grupo foi escolhida uma única participante, cada grupo foi direcionado, propositalmente, para caminhos diferentes: "câncer" e "corpo saudável". As duas participantes vendadas foram colocadas distantes dos dois caminhos, sem a noção da direção dos caminhos, foram orientadas e guiadas através de ajuda das outras participantes a seguir pelo caminho. Essa etapa possibilitou uma reflexão sobre o caminho que percorremos e sua consequência, sendo uma forma de demonstrar que se seguirmos hábitos saudáveis e exames de prevenção regular da doença termos uma vida mais saudável, no entanto, se não aderirmos à realização do PCCU, 
por qualquer motivo, podemos, consequentemente, evoluir para o câncer; c) a terceira etapa consistiu em uma roda de conversa a fim de um diálogo aberto e exposição de dúvidas sobre o assunto. Ao final das ações, houve entrega de brindes e oferecido um lanche às participantes como forma de incentivo à participação.

Dentre as 250 mulheres que preencheram o formulário, 165 participaram das ações educativas, sendo 95/100 moradoras da zona urbana e 70/150 residentes na área urbana. Acredita-se que o ideal seria um número maior de participantes, visto a necessidade identificada pautada nos resultados deste estudo. No entanto, o não comparecimento de algumas já era previsto após perceber a dificuldade de acesso que essas mulheres enfrentam para se deslocarem, especialmente as residentes nas áreas rurais.

Durante as rodas de conversa, as participantes relataram a importância da ação educativa e esclarecimentos quanto a necessidade em realizar o exame, sentindo-se dispostas e decididas a quebrar as barreiras em busca de um corpo saudável.

Discussão

Neste estudo foram incluídas mulheres entre 20 e 25 anos, tendo em vista o início precoce da atividade sexual e a incidência do HPV nas adolescentes, conforme aponta Hugo et al. [8] em seu estudo, no qual $33 \%$ das mulheres entrevistadas haviam tido relações sexuais antes dos 15 anos, afirmando também, que essa precocidade com que está ocorrendo o início da vida sexual, pode ser um agravo à saúde.

Quanto à escolaridade, Silva et al. [9,10] afirmam que a baixa escolaridade é um fator que propicia a não realização do exame PCCU e, consequentemente, aumenta a probabilidade de desenvolver o câncer do colo do útero, seus estudos relatam forte associação entre alteração celular epitelial e escolaridade inferior ao ensino fundamental completo.

Segundo o IBGE [6], em Portel o salário médio mensal da população é de 2,1 salários mínimos e a proporção de pessoas ocupadas em relação à população total é de $7,1 \%$, bem abaixo dos outros municípios do estado e outras cidades do país todo, além de domicílios com rendimentos mensais de até meio salário mínimo por pessoa, com $51 \%$ da população nessas condições, revelando o porquê do município de Portel aparecer nas estatísticas como um dos munícipios com índice de Desenvolvimento Humano (IDH) mais baixo do Pará e do Brasil. O perfil socioeconômico das mulheres de Portel as coloca em situação de vulnerabilidade ao câncer do colo do útero, pois tanto a baixa escolaridade quanto a baixa renda são fatores que propiciam a não realização do exame PCCU e, consequentemente, aumentam a probabilidade do desenvolvimento da doença $[9,11]$.

O maior percentual de mulheres da área urbana adstrita em relação às da zona rural que realizaram o PCCU pode ter como motivo a dificuldade de acesso das mulheres residentes na zona rural, uma vez que este exame é realizado nos postos de saúde localizados na área urbana do município. Para Davim et al. [12], fatos como esse pode nos remeter a ideia de que, mesmo sendo um procedimento de baixo custo, a coleta do PCCU não está incorporada a todos os serviços de saúde, tendo utilização reduzida e não disponível a toda população feminina.

Um fator relevante neste estudo é que embora a maioria das participantes já tenha realizado o PCCU, muitas delas não o estão realizando de acordo com o preconizado pelo Ministério da Saúde, que recomendada a repetição do Papanicolau a cada três anos, após dois exames normais consecutivos realizados com intervalo de um ano [13].

Segundo Souza e Alves [14], os motivos da não realização do PCCU estão associados a fatores como: desconhecimento do próprio corpo, desconhecimento da importância do exame e de sua realização, dificuldade de acesso, demanda reprimida, falta de oportunidade que a usuária tem para falar sobre si e sobre sua sexualidade, como também, pelo desconhecimento sobre o próprio câncer, acrescido de sentimentos causadores de incômodo, medo e vergonha, tabus e ideias preconceituosas sobre a mulher. Em um outro estudo identificou-se que as mulheres mais vulneráveis a desenvolver o câncer de colo de útero concentravam-se onde as barreiras geográficas e de acesso ao serviço de saúde eram maiores, corroborando os resultados deste estudo [15].

Sobre a falta de interesse em realizar o PCCU, está relacionado ao fato de muitas mulheres tratarem o câncer de colo do útero como uma doença distante do seu contexto de vida, favorecendo a falta de atitude e a não realização do exame e que, na maioria das vezes, esse conhecimento consensual só é mudado quando elas mesmas ou pessoas próximas são acometidas pela doença [16]. 
Nota-se que uma grande parte da população ainda não tem um conhecimento adequado sobre o verdadeiro objetivo do exame [17], o que pode também influenciar o sentimento de constrangimento no momento da coleta. A vergonha torna-se uma barreira essencial para realização do exame e pode causar até descontinuidade da assistência, pois a exposição do corpo durante o procedimento do exame é algo intenso para a mulher, as coloca em situação de vulnerabilidade, sendo exposta ao toque, manipulação e julgamento do seu corpo por outra pessoa, além da impotência, desproteção e perda do domínio do corpo que a posição ginecológica proporciona $[18,19]$.

O sentimento do medo, também mencionado pelas participantes deste estudo, faz com que algumas mulheres adiem a realização do preventivo, revelando a falta de informações sobre a importância do diagnóstico precoce, probabilidade de cura mais elevada e tratamentos mais sutis [20]. Assim sendo, existe a necessidade de se esclarecer quanto à importância da realização do exame preventivo, pois a adesão das mulheres à sua realização está intimamente relacionada ao grau de conhecimento das mesmas [16].

Avaliou-se o resultado deste estudo como positivo, pela abrangência das ações educativas entre as mulheres do município de Portel. Acredita-se que a mulher, como principal beneficiária das ações contra o câncer de colo uterino, deve ser esclarecida sobre todas as etapas do PCCU e o profissional enfermeiro capacitado pode atuar junto a equipe multiprofissional e ser um elo entre a população e o serviço de saúde.

Oliveira et al. [21] evidenciam a importância do papel da Estratégia Saúde da Família (ESF) por representar um modelo de atenção voltada para a prevenção, promoção, proteção e recuperação da saúde com o objetivo de criar vínculo entre equipe de saúde e usuário. Dessa forma, há maior facilidade de acesso, comunicação e troca de informações com a população tirando dúvidas, medo e anseios e proporcionando conhecimento adequado acerca de se fazer a prevenção, além de facilitar o rastreamento de mulheres para que façam o exame rotineiro de PCCU.

Neste estudo, durante as ações educativas, houve bastante participação das mulheres do estudo, questionando e partilhando suas experiências pessoas, bem como, sanando as dúvidas e fazendo comentários a respeito de tudo o que foi exposto e vivenciado. Essas mulheres relataram que foi importante participar do estudo, pois puderam conhecer melhor o tema, sendo fundamental para a reflexão pessoal. Além disso, algumas pontuaram as necessidades em geral e de melhoria, como incentivo e investimento do governo para se cumprir as ações.

Entende-se a participação dos ACS neste estudo como de suma importância, pelo quantitativo expressivo de mulheres participantes, obtidos pela busca ativa realizada. Neste contexto, esse profissional, dentro da ESF, tem um papel fundamental, pois além de acompanhar a história de saúdeldoença da comunidade, ele traz as inúmeras demandas enfrentadas por ela nesse processo [22]. Entretanto, há a necessidade de vínculo entre os profissionais da saúde e a população, sendo o ACS um possível elo que possibilita a confiança e a relação entre a equipe de saúde e a comunidade assistida.

Contudo, percebe-se a importância de todos os profissionais da ESF para que, não só a busca ativa seja realizada efetivamente, mas que toda a assistência relacionada à prevenção do câncer de colo uterino, como a coleta de informações e do material para exame, bem como orientações a toda população, não apenas às mulheres na faixa etária preconizada pelo Ministério da Saúde, mas homens, adolescentes, para desmistificar o procedimento e para informar sobre a importância na realização do PCCU e seu seguimento adequado.

Os motivos relatados pelas participantes, residentes na zona rural e urbana de Portel, para não-adesão ao PCCU foram compatíveis com as descritas em outros estudos científicos, sendo os principais: dificuldade de acesso ao local de realização do exame e medo, vergonha/constrangimento para coletar o material, falta de interesse, não saber que deveria realizar o exame, falta de material e a proibição do marido.

O estudo possibilitou demonstrar a importância na adesão ao PCCU, através de ações educativas, desenvolvidas e pensadas de acordo com as justificativas apresentadas pelas mulheres para a não realização do exame.

Compreendemos que o enfermeiro, como membro da equipe de saúde e coordenador da ESF, tem um papel fundamental ao perceber a realidade e dificuldade da população de sua área de abrangência, possibilitando a criação de estratégias que visem minimizar os problemas 
e, especificamente, auxiliando na detecção precoce tanto de lesões precursoras quanto do próprio câncer do colo uterino.

Estudos deste tipo poderão servir como modelo e prática a serem realizados em outras unidades de saúde de Portel e para as demais cidades da região, pela similaridade nas realidades periféricas e rurais, servindo como base epidemiológica para a atuação do gestor de saúde. Isso pode ser confirmado ao nos depararmos com a intensificação de ações, realizadas pela Secretaria de Saúde de Portel, após a realização deste estudo, como ações de busca ativa e campanhas de coleta do PCCU, culminando em um aumento na demanda e realização do exame pelas mulheres do município.

\section{Referências}

1. Brasil. Ministério da Saúde. Secretaria de Vigilância em Saúde. Departamento de Vigilância das Doenças Transmissíveis. Manual de normas e procedimentos para vacinação. Brasília: Ministério da Saúde; 2014. [citado 2018 Jan 5]. Disponível em: http://bvsms.saude.gov.br/bvs/publicacoes/manual procedimentos vacinacao.pdf

2. Inca. Instituto Nacional de Controle de Câncer. Estimativa 2018: incidência de câncer no Brasil. Rio de Janeiro: Inca; 2018. [citado 2018 Abril 22]. Disponível em: https://www.inca.gov.br/sites/ufu.sti.inca.local/files//media/document//estimativaincidencia-de-cancer-no-brasil-2018.pdf

3. Aguilar RP, Soares DA. Barreiras à realização do exame Papanicolau: perspectivas de usuárias e profissionais da estratégia de saúde da família da cidade de Vitória da Conquista/BA. Physis Rev Saúde Coletiva 2015;25(2):359-79. https://doi.org/10.1590/S0103-73312015000200003

4. Carvalho RS, Nunes RMV, Oliveira JD, Davim RMB, Rodrigues ESRC, Menezes PCM. Perfil preventivo do câncer de colo uterino em trabalhadoras da Enfermagem. Revista de Enfermagem UFPE Online 2017;11(6):2257-63. https://doi.org/10.5205/reuol.1082796111-1-ED.1106201701

5. Casarin MR, Piccoli JCE. Educação em saúde para prevenção do câncer de colo do útero em mulheres do município de Santo Ângelo/RS. Ciênc Saúde Coletiva 2011;16(9):3925-32.

6. IBGE. Instituto Brasileiro de Geografia e Estatística. Indicadores econômicos municipais: uma análise dos resultados do universo do censo demográfico 2017. Rio de Janeiro: IBGE; 2018. [citado 2018 Ago 15]. Disponível em:

https://cidades.ibge.gov.br/brasil/pa/portel/panorama

7. Andrade MS, Almeida MMG, Araújo TM, Santos KOB. Fatores associados a nãoadesão ao Papanicolau entre mulheres atendidas pela Estratégia Saúde da Família em Feira de Santana - Bahia, 2010. Epidemiol Serv Saúde 2014;23(1):111-20.

8. Hugo TDO, Maier VT, Jansen K, Rodrigues CEG, Cruzeiro ALS, Ores LC, Pinheiro RT, Silva R, Souza LDM et al. Fatores associados à idade da primeira relação sexual em jovens: estudo de base populacional. Cad Saúde Pública 2011;27(11):2207-14. https://doi.org/10.1590/S0102-311X2011001100014

9. Silva MASA, Teixeira BEM, Ferrari RAP, Cestari MEW, Carteli AAM. Fatores relacionados a não-adesão a realização do exame de Papanicolau. Rev Rene 2015;16(4);532-9. [citado 2018 Set 10]. Disponível em: http://periodicos.ufc.br/rene/article/view/2745/2128

10. Silva AM, Guedes GW, Dantas AFLS, Nóbrega MM. Perfil epidemiológico do câncer do colo do útero na Paraíba 2016;16(4):2447-31.

11. Melo SCCS, Prates L, Carvalho MDB, Marcon SS, Pelloso SM. Alterações citopatológicas e fatores de risco para a ocorrência do câncer de colo uterino. Rev Gaúcha de Enferm 2009;30(4):602-8. https://doi.org/10.1590/S198314472009000400004

12. Davim RMB, Torres GV, Silva RAR, Silva DAR. Conhecimento de mulheres de uma unidade básica de saúde da cidade de Natal/RN sobre o exame de Papanicolau. Rev Esc Enferm USP 2005;39(3):296-302. https://doi.org/10.1590/S008062342005000300007

13. Brasil. Ministério da Saúde. Secretaria de Atenção à Saúde. Departamento de Atenção Básica. Controle dos cânceres do colo do útero e da mama. Brasília: Ministério da Saúde; 2013. [citado 2018 Jan 20]. Disponível em: http://bvsms.saude.gov.br/bvs/publicacoes/controle canceres colo utero 2013.pdf 
14. Souza G, Alves PS. Estratégias educativas para prevenção e redução da morbimortalidade do câncer do colo uterino. Revista Saúde e Pesquisa 2015;8(2):31726. [citado 2018 Set 10]. Disponível em: file:///C:/Users/user/Downloads/4258-17473-1PB.pdf

15. Ferreira MLSM. Motivos que influenciam a não realização do exame de Papanicolaou segundo a percepção de mulheres. Esc Anna Nery [Online] 2009;13(2):378-84. https://doi.org/10.1590/S1414-81452009000200020

16. Albuquerque VR, Miranda RV, Leite CA, Leite MCA. Exame preventivo do câncer de câncer de colo do útero: conhecimento de mulheres. Rev Enferm UFPE on line 2016;10(5):4208-18.

17. Vasconcelos CTM. Efeitos de uma intervenção educativa na adesão das mulheres à consulta de retorno para receber o exame Papanicolaou [Dissertação]. Fortaleza/CE: Universidade Federal Do Ceará. Programa De Pós-Graduação em Enfermagem; 2012.

18. Rafael RMR, Moura ATMS. Barreiras na realização da colpocitologia oncótica: um inquérito domiciliar na área de abrangência da saúde da família de Nova Iguaçu, Rio de Janeiro, Brasil. Cad Saúde Pública 2010;26(5):1045-50. https://doi.org/10.1590/S0102311X2010000500026

19. Souza GD, Oliveira RAA, Stevanin A, Sousa MF, Almeida EC. A concepção das mulheres de Mirandópolis, São Paulo, acerca do exame de Papanicolau. Rev Enferm UFSM 2013;3(3):470-9. https://doi.org/10.5902/217976929647

20. Soares MC, Mishima SM, Meincke SMK, Simino GPR. Câncer de colo uterino: caracterização das mulheres em um município do sul do Brasil. Esc Anna Nery 2010;14(1):90-96. https://doi.org/10.1590/S1414-81452010000100014

21. Oliveira WMA, Barbosa MA, Mendonça BOM, Silva AA, Santos LCF, Nascimento LCD. Adesão de mulheres de 18 a 50 anos ao exame colpocitológico na estratégia saúde da família. Rev Enf Ref 2012;3(7):15-22. https://doi.org/10.12707/RIIl11139

22. Junges JR, Mazari CK, Selli L. Agentes comunitários de saúde: perfil e formação. Ciênc Saúde Coletiva 2008. https://doi.org/10.1590/S1413-81232011000700019 